\title{
肉桂酸-香豆素酯类似物的合成及抑制酪氨酸酶活性研究
}

\author{
林芷晴 $a$ \\ 夏婉铃 ${ }^{a}$ 刘仁义 ${ }^{a}$ 姜少华*,a
$\left({ }^{a}\right.$ 五邑大学生物科技与大健康学院
广东江门 529000)
$\left({ }^{b}\right.$ 华南理工大学化学与化工学院 \\ 马志强 $*, a, b$
}

\begin{abstract}
摘要 在药物化学中, 天然产物结构修饰和骨架杂合策略是拓展骨架生物学活性和寻找高活性先导化合物的重要途 径. 以取代肉桂酸和羟基香豆素为原料，合成了两个系列的肉桂酸-香豆素酯类似物，并评估了合成化合物对酪氨酸酶 的抑制活性. 结果表明, 肉桂酸-香豆素类似物均有较好的抑制酪氨酸酶活性, 其中 2-氧代- $2 H$-苯并吡喃-4-基 $(E)$-3-(4着基苯基)丙烯酯 $\left(\mathbf{C}_{\mathbf{8}}\right)$ 和 2-氧代- $2 H$-苯并吡喃-7-基( $E$ )-3-(4-羊基苯基)丙烯酯 $\left(\mathbf{D}_{\mathbf{8}}\right.$ )抑制酪氨酸酶的活性最强, $\mathrm{IC}_{50}$ 分别为 $(10.7 \pm 0.7)$ 和 $(2.2 \pm 0.2) \mu \mathrm{mol} \cdot \mathrm{L}^{-1}$, 分别是曲酸 $\left[\mathrm{IC}_{50}:(28.5 \pm 1.1) \mu \mathrm{mol} \cdot \mathrm{L}^{-1}\right]$ 的 3 倍和 13 倍. 构效关系分析结果显示, 3 个 取代基 $(\mathrm{F}, \mathrm{Cl}, \mathrm{OH})$ 的引入可显著提高化合物的酪氨酸酶抑制活性，取代肉桂酸与 7-羟基香豆素缩合产物的活性高于其 与 4-着基香豆素缩合产物. 动力学研究表明, 化合物 $\mathbf{C}_{\mathbf{8}}$ 和 $\mathbf{D}_{\mathbf{8}}$ 对酪氨酸酶的抑制是一种可逆、混合型的抑制作用. $\mathbf{C}_{\mathbf{8}}$ 和 $\mathbf{D}_{\mathbf{8}}$ 的 $K_{\mathrm{I}}$ 分别为 $1.07,20.61 \mu \mathrm{mol} \cdot \mathrm{L}^{-1}, K_{\mathrm{IS}}$ 分别为 $3.72,27.09 \mu \mathrm{mol} \cdot \mathrm{L}^{-1}$. 最后, 采用分子对接模拟了化合物 $\mathbf{C}_{\mathbf{8}}$ 和 $\mathbf{D}_{\mathbf{8}}$ 与 酪氨酸酶的结合情况.
\end{abstract}

关键词 酪氨酸酶; 香豆素; 肉桂酸; 分子对接

\section{Synthesis of Cinnamic Acid-Coumarin Ester Analogs and Inhibition of Tyrosinase Activity}

\author{
Lin, Zhiqing ${ }^{a} \quad$ Xia, Wanling $^{a} \quad$ Liu, Renyi $^{a} \quad$ Jiang, Shaohua $^{*, a} \quad$ Ma, Zhiqiang ${ }^{*, a, b}$ \\ ( ${ }^{a}$ School of Biotechnology and Grand Health, Wuyi University, Jiangmen, Guangdong 529000) \\ ( ${ }^{b}$ School of Chemistry \& Chemical Engineering, South China University of Technology, Guangzhou 510641)
}

\begin{abstract}
In medicinal chemistry, the structural modification of natural product and skeleton hybridization strategies is important way to improve the biological activity of template compound and find highly active lead compounds. In this work, two series of cinnamic acid-coumarin ester analogs were synthesized by using cinnamic acid and hydroxycoumarin as raw materials. And the tyrosinase inhibitory activity of the synthesized compounds was evaluated. The results indicated that the cinnamic acid-coumarin analogs had favourable tyrosinase inhibitory activity, especially 2-oxo-2H-benzopyran-4-yl $(E)$-3-(4hydroxyphenyl)propenyl ester $\left(\mathbf{C}_{\mathbf{8}}\right)$, 2-oxo-2H-benzopyran-7-yl(E)-3-(4-hydroxyphenyl)propenyl ester and $\left(\mathbf{D}_{\mathbf{8}}\right)$ with $\mathrm{IC}_{50}$ of $(10.7 \pm 0.7)$ and $(2.2 \pm 0.2) \mu \mathrm{mol} \cdot \mathrm{L}^{-1}$, respectively, which are 3 and 13 times that of kojic acid $\left(\mathrm{IC}_{50}(28.5 \pm 1.1) \mu \mathrm{mol} \cdot \mathrm{L}^{-1}\right)$. The structure-activity relationship analysis results showed that the introduction of substituents like $\mathrm{F}, \mathrm{Cl}$, and $\mathrm{OH}$ could efficiently enhance the tyrosinase inhibitory activity, and the inhibitory activity of condensation product of substituted cinnamic acid with 7-hydroxycoumarin was higher than that of substituted cinnamic acid with 4-hydroxycoumarin. Kinetic studies showed that the inhibitions of tyrosinase by compounds $\mathbf{C}_{\mathbf{8}}$ and $\mathbf{D}_{\mathbf{8}}$ are reversible mixed-type inhibitory effects. $K_{\mathrm{I}}$ values of $\mathbf{C}_{\mathbf{8}}$ and $\mathbf{D}_{8}$ were 1.07 and $20.61 \mu \mathrm{mol} \cdot \mathrm{L}^{-1}$, respectively, and $K_{\mathrm{IS}}$ values were 3.72 and $27.09 \mu \mathrm{mol} \cdot \mathrm{L}^{-1}$, respectively. Finally, molecular docking was carried out to simulate the docking between compounds $\mathbf{C}_{\mathbf{8}}$ and $\mathbf{D}_{\mathbf{8}}$ with tyrosinase.

Keywords tyrosinase; coumarin; cinnamic acid; molecular docking
\end{abstract}

酪氨酸酶(EC 1.14.18.1)是一种含铜离子的多酚氧 化酶, 广泛分布于动植物和微生物中 ${ }^{[1-2]}$. 它参与组织黑 色素的生物合成过程, 是黑色素的限速酶 ${ }^{[3]}$. 在黑色素
生成过程中，酪氨酸酶将 $L$-酪氨酸催化氧化为 $L-3,4-二$ 羟基苯丙氨酸 $(L-\mathrm{DOPA})$, 并进一步将 $L-\mathrm{DOPA}$ 氧化为邻 醌, 最终生成黑色素 ${ }^{[4-5]}$. 若黑色素过分积累, 则会引起

* Corresponding authors. E-mail: wyuchemjsh@126.com; 23090330@qq.com.

Received May 4, 2020; revised May 29, 2020; published online June 19, 2020

Project supported by the Department of Education of Guangdong Province (Nos. 2017KSYS010, 2017KZDXM084, 2019KZDZX2003, 19KZDXM035).

广东省教育厅基金(Nos. 2017KSYS010, 2017KZDXM084, 2019KZDZX2003, 2019KZDXM035)资助项目. 
色素沉着, 并导致各种色斑甚至黑色素瘤 ${ }^{[6-8]}$. 酪氨酸酶 抑制剂可降低酪氨酸酶的催化速率, 从而预防黑色素的 异常沉积. 当前, 酪氨酸酶抑制剂可用于预防或治疗黄 褐斑、雀斑等色素沉着疾病及美白产品 ${ }^{[9-12]}$. 此外, 酪氨 酸酶抑制剂还可用作食品保鲜剂, 有效预防食品酶促褐 变而导致的变质等问题 ${ }^{[13-15]}$. 虽然当前已发现较多的酪 氨酸酶抑制剂, 但仅曲酸、苯二酚等有实际的应用, 实 践研究还发现曲酸、苯二酚等具有引发细胞毒性、皮肤 癌、皮炎等副作用 ${ }^{[16-20]}$. 这些都激励我们开发新型、高 效、低毒的酪氨酸酶抑制剂.

肉桂酸是肉桂、安息香等天然药用植物的重要活性 成分，肉桂酸及其衍生物不仅具有抗病毒、抗微生物、 杀真菌、抗癌和抗氧化等药理作用, 并具有一定的酪氨 酸酶抑制作用 ${ }^{[21-24]}$. 香豆素是天然产物中非常重要的一 类具有苯并吡喃骨架的化合物, 许多研究显示香豆素及 其衍生物有着较强的抗炎、抗癌、抗氧化、抗肿瘤及抑 制酪氨酸酶等作用 ${ }^{[25-28]}$.

天然产物结构修饰是寻找较高活性先导化合物的 重要途径, 骨架杂合策略是拓展其生物学活性, 提升药 理作用，克服耐药性等的关键策略. 当前, 很多临床药 物和高活性分子都是由结构修饰或者骨架杂合策略获 得. 因此, 我们在课题组前期研究的基础上 ${ }^{[29-33]}$, 将具 有优良活性的天然产物分子肉桂酸和香豆素杂合, 合成 了两个系列的肉桂酸-香豆素酯类似物 $\left(\mathbf{C}_{\mathbf{1}} \sim \mathbf{C}_{\mathbf{9}}\right.$ 和 $\mathbf{D}_{\mathbf{1}} \sim$ $\left.\mathbf{D}_{9}\right)$, 并评估了合成化合物的酪氨酸酶的抑制活性, 以 期发现高活性的先导化合物.

\section{1 结果与讨论}

\section{1 肉桂酸-香豆素酯类似物的合成}

肉桂酸-香豆素酯类似物 $\left(\mathbf{C}_{1} \sim \mathbf{C}_{9}\right.$ 和 $\left.\mathbf{D}_{1} \sim \mathbf{D}_{9}\right)$ 的合成 路线如 Scheme 1 所示. 取代肉桂酸在二氯亚砜作用下 生成对应的酰氯，与 4-羟基香豆素或 7-羟基香豆素反应 分别得到对应的目标化合物 $\mathbf{C}_{\mathbf{1}} \sim \mathrm{C}_{\mathbf{9}}$ 和 $\mathbf{D}_{\mathbf{1}} \sim \mathbf{D}_{\mathbf{9}}$. 文中涉 及的化合物均为已知化合物 ${ }^{[34-35]}$, 且化合物结构均经 NMR, IR, MS 确认.

\section{2 化合物对酪氨酸酶的抑制活性评估}

以 $L$-多巴为底物, 测定了化合物 $\mathbf{C}_{\mathbf{1}} \sim \mathbf{C}_{\mathbf{9}}$ 和 $\mathbf{D}_{\mathbf{1}} \sim \mathbf{D}_{\mathbf{9}}$ 对蘑菇酪氨酸酶的抑制活性(表 1). 首先笁选了肉桂酸香豆素酯类似物的终浓度为 $200 \mu \mathrm{mol} \cdot \mathrm{L}^{-1}$ 时酪氨酸酶 的抑制活性, 发现化合物 $\mathbf{C}_{\mathbf{1}} \sim \mathbf{C}_{\mathbf{9}}$ 和 $\mathbf{D}_{\mathbf{1}} \sim \mathbf{D}_{\mathbf{9}}$ 都有一定的 酪氨酸酶的抑制作用, 其中 $\mathbf{C}_{4}, \mathbf{C}_{5}, \mathbf{C}_{8}, \mathbf{D}_{\mathbf{4}}, \mathbf{D}_{5}, \mathbf{D}_{\mathbf{8}}$ 表现出 较明显的抑制酪氨酸酶作用，抑制作用大于 $40 \%$ ，而其 他化合物在 $200 \mu \mathrm{mol} \cdot \mathrm{L}^{-1}$ 时抑制酪氨酸酶的作用低于 $40 \%$. 接着测定了化合物 $\mathbf{C}_{\mathbf{4}}, \mathbf{C}_{5}, \mathbf{C}_{\mathbf{8}}, \mathbf{D}_{\mathbf{4}}, \mathbf{D}_{5}, \mathbf{D}_{\mathbf{8}}$ 抑制酪氨 酸酶的半数抑制浓度 $\left(\mathrm{IC}_{50}\right)$, 同时也测定了母体化合物

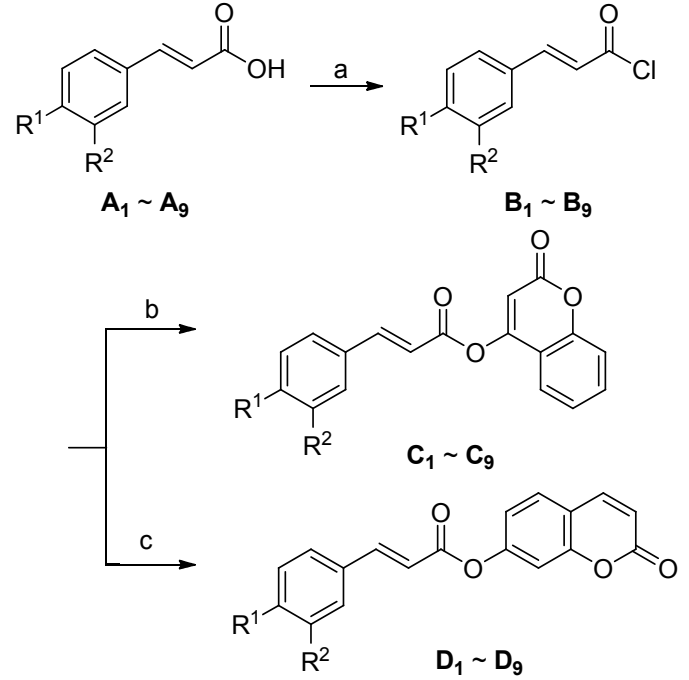

$A_{1}, R^{1}=H, R^{2}=H ; A_{2}, R^{1}=C_{3}, R^{2}=H ; A_{3}, R^{1}=\mathrm{OCH}_{3}, R^{2}=H ;$ $\mathbf{A}_{4}, \mathrm{R}^{1}=\mathrm{F}, \mathrm{R}^{2}=\mathrm{H} ; \mathbf{A}_{5}, \mathrm{R}^{1}=\mathrm{Cl}, \mathrm{R}^{2}=\mathrm{H} ; \mathbf{A}_{6}, \mathrm{R}^{1}=\mathrm{Br}, \mathrm{R}^{2}=\mathrm{H}$; $A_{7}, R^{1}=C_{3}, R^{2}=H ; A_{8}, R^{1}=O H, R^{2}=H ; A_{9}, R^{1}=O H, R^{2}=O_{3}$ Reagents and conditions: (a) $\mathrm{SOCl}_{2}$, DMF, DCM, room temperature, $6 \mathrm{~h}$; (b) 4-Hydroxycoumarin, DIEA, DCM, $0{ }^{\circ} \mathrm{C}$; (c) 7-Hydroxycoumarin, DIEA, DCM, $0^{\circ} \mathrm{C}$

图式 1 化合物 $\mathrm{C}_{\mathbf{1}} \sim \mathrm{C}_{\mathbf{9}}$ 和 $\mathrm{D}_{\mathbf{1}} \sim \mathrm{D}_{\mathbf{9}}$ 的合成路线 Scheme 1 Synthetic route of compounds $C_{1} \sim C_{9}$ and $D_{1} \sim D_{9}$

表 1 化合物对酪氨酸酶活性的抑制作用

Table 1 Inhibition of compounds on tyrosinase activity

\begin{tabular}{clll}
\hline Number & \multicolumn{1}{c}{$\mathrm{R}^{1}$} & $\mathrm{R}^{2}$ & $\mathrm{IC}_{50} /\left(\mu \mathrm{mol} \cdot \mathrm{L}^{-1}\right)$ \\
\hline $\mathbf{C}_{\mathbf{1}}$ & $\mathrm{H}$ & $\mathrm{H}$ & $>200^{a}$ \\
$\mathbf{C}_{\mathbf{2}}$ & $\mathrm{CH}_{3}$ & $\mathrm{H}$ & $>200^{a}$ \\
$\mathbf{C}_{\mathbf{3}}$ & $\mathrm{OCH}_{3}$ & $\mathrm{H}$ & $>200^{a}$ \\
$\mathbf{C}_{\mathbf{4}}$ & $\mathrm{F}$ & $\mathrm{H}$ & $112.2 \pm 3.4$ \\
$\mathbf{C}_{\mathbf{5}}$ & $\mathrm{Cl}$ & $\mathrm{H}$ & $105.5 \pm 2.8$ \\
$\mathbf{C}_{\mathbf{6}}$ & $\mathrm{Br}$ & $\mathrm{H}$ & $>200^{a}$ \\
$\mathbf{C}_{\mathbf{7}}$ & $\mathrm{CF}_{3}$ & $\mathrm{H}$ & $>200^{a}$ \\
$\mathbf{C}_{\mathbf{8}}$ & $\mathrm{OH}$ & $\mathrm{H}$ & $10.7 \pm 0.7$ \\
$\mathbf{C}_{9}$ & $\mathrm{OH}$ & $\mathrm{OCH}$ & $>200^{a}$ \\
肉桂酸 & & & $201.4 \pm 5.3$ \\
曲酸 & & & $28.5 \pm 1.1$ \\
$\mathbf{D}_{\mathbf{1}}$ & $\mathrm{H}$ & $\mathrm{H}$ & $>200^{a}$ \\
$\mathbf{D}_{\mathbf{2}}$ & $\mathrm{CH}_{3}$ & $\mathrm{H}$ & $>200^{a}$ \\
$\mathbf{D}_{\mathbf{3}}$ & $\mathrm{OCH}$ & $\mathrm{H}$ & $>200^{a}$ \\
$\mathbf{D}_{\mathbf{4}}$ & $\mathrm{F}$ & $\mathrm{H}$ & $78.4 \pm 3.2$ \\
$\mathbf{D}_{\mathbf{5}}$ & $\mathrm{Cl}$ & $\mathrm{H}$ & $76.5 \pm 3.1$ \\
$\mathbf{D}_{\mathbf{6}}$ & $\mathrm{Br}$ & $\mathrm{H}$ & $>200^{a}$ \\
$\mathbf{D}_{7}$ & $\mathrm{CF}$ & $\mathrm{H}$ & $>200^{a}$ \\
$\mathbf{D}_{\mathbf{8}}$ & $\mathrm{OH}$ & $\mathrm{H}$ & $2.2 \pm 0.2$ \\
$\mathbf{D}_{\mathbf{9}}$ & $\mathrm{OH}$ & $\mathrm{OCH}$ & $>200^{a}$ \\
香豆素 & & & $>200^{a}$ \\
\hline
\end{tabular}

${ }^{a}$ 化合物浓度 $200 \mu \mathrm{mol} \cdot \mathrm{L}^{-1}$ 时, 酪氨酸酶抑制活性低于 $40 \%$.

肉桂酸，香豆素及阳性对照曲酸的抑制酪氨酸酶活性. 结果显示：肉桂酸和香豆素显示出中等强度的酪氨酸酶 抑制活性, $\mathrm{IC}_{50}$ 均高于 $200 \mu \mathrm{mol} \cdot \mathrm{L}^{-1}$; 而酯化产物 $\mathbf{C}_{\mathbf{4}}, \mathbf{C}_{\mathbf{5}}$, $\mathbf{C}_{8}, \mathbf{D}_{4}, \mathbf{D}_{5}, \mathbf{D}_{\mathbf{8}}$ 显示出了较强的活性, $\mathrm{IC}_{50}$ 值 $(2.2 \pm 0.2) \sim$ 
(112.2 \pm 3.4$) \mu \mathrm{mol} \cdot \mathrm{L}^{-1}$, 抑制活性明显高于母体化合物. 其中 $\mathbf{C}_{8}$ 和 $\mathbf{D}_{8}$ 抑制酪氨酸酶活性显著高于阳性对照曲酸 $\left[\mathrm{IC}_{50}=(28.5 \pm 1.1) \mu \mathrm{mol} \cdot \mathrm{L}^{-1}\right]$. 结果提示: 取代肉桂酸和 香豆素杂合后, 改善了骨架的生物活性, 能够更好地与 酪氨酸酶结合, 进而获得更高酪氨酸酶抑制活性的化合 物.

接着对化合物抑制酪氨酸酶活性的构效关系进行 分析. 比较两个系列化合物 $\mathbf{C}_{\mathbf{1}} \sim \mathbf{C}_{\mathbf{9}}$ 和 $\mathbf{D}_{\mathbf{1}} \sim \mathbf{D}_{\mathbf{9}}$ 的酪氨酸 酶抑制活性, 发现 7-差基香豆素杂合产物 $\left(\mathbf{D}_{1} \sim \mathbf{D}_{9}\right)$ 较 4羟基香豆素杂合产物 $\left(\mathbf{C}_{\mathbf{1}} \sim \mathbf{C}_{\mathbf{9}}\right)$ 有更好的酪氨酸酶抑制活 性. 接着以 $\mathrm{D}_{1}$ 为母体化合物, 分析 $\mathrm{D}_{\mathbf{1}} \sim \mathrm{D}_{9}$ 的活性数据, 可得到肉桂酸取代基对化合物抑制酪氨酸酶活性的影 响, 结果显示: 肉桂酸苯环 4 位取代基化合物对酪氨酸 酶抑制活性有显著影响. 化合物 $\mathbf{D}_{\mathbf{8}}\left[\mathrm{IC}_{50}=(2.2 \pm 0.2)\right.$ $\left.\mu \mathrm{mol} \cdot \mathrm{L}^{-1}\right]$ 的抑制作用最大, $\mathbf{D}_{5}\left[\mathrm{IC}_{50}=(76.5 \pm 3.1)\right.$ $\left.\mu \mathrm{mol} \cdot \mathrm{L}^{-1}\right], \mathbf{D}_{4}\left[\mathrm{IC}_{50}=(78.4 \pm 3.2) \mu \mathrm{mol} \cdot \mathrm{L}^{-1}\right]$ 的活性次之, 而其他取代基团的 $\mathrm{IC}_{50}$ 值都高于 $200 \mu \mathrm{mol} \cdot \mathrm{L}^{-1}$, 这一结 果提示 $\mathrm{Cl}, \mathrm{F}, \mathrm{OH}$ 基团的引入可有效增强化合物酪氨酸 酶抑制活性, 而 $\mathrm{Br}, \mathrm{OCH}_{3}, \mathrm{CF}_{3}, \mathrm{CH}_{3}$ 对活性的增加无益. 系列化合物 $\mathbf{D}_{\mathbf{1}} \sim \mathbf{D}_{\mathbf{9}}$ 的构效关系结果与化合物系列 $\mathbf{C}_{\mathbf{1}} \sim$ $\mathbf{C}_{9}$ 基本一致. 化合物 $\mathbf{C}_{8}$ 和 $\mathbf{D}_{8}$ 的高活性可能与化合物分 子中羟基可与酪氨酸酶间形成更强的作用力有关. 因此 在后期化合物的设计合成中，应重点考虑 4-差基肉桂酸 杂合 7 位香豆素骨架的进一步修饰.

\section{3 化合物抑制酪氨酸酶的机理}

以抑制酪氨酸酶活性最佳的化合物 $\mathbf{C}_{8}$ 和 $\mathbf{D}_{8}$ 为代表 化合物, 探讨了肉桂酸-香豆素酯类似物抑制酪氨酸酶 的机理. 酶的抑制类型主要为可逆与不可逆两种, 可以 通过不同浓度抑制剂作用下的酶促反应的速率与酶浓 度的曲线来确定. 如图 1 所示, 在不同浓度的化合物 $\mathbf{C}_{\mathbf{8}}$ 和 $\mathbf{D}_{\mathbf{8}}$ 作用下, 酶促反应速率与酶浓度曲线都交于原点, 并且随着抑制剂浓度的增加, 曲线斜率降低. 根据酶的 抑制类型的定义, 可知化合物 $\mathbf{C}_{\mathbf{8}}$ 和 $\mathbf{D}_{\mathbf{8}}$ 是一种可逆的酪 氨酸酶抑制剂 ${ }^{[10,26]}$.

接着采用 Lineweaver-Burk 双倒数曲线分析了化合 物 $\mathbf{C}_{\mathbf{8}}$ 和 $\mathbf{D}_{\mathbf{8}}$ 的抑制剂类型. 抑制剂类型主要包含竞争性 抑制剂、非竞争性抑制剂、反竞争性抑制剂、混合型抑 制剂几种，可通过不同浓度抑制剂作用下的酶促反应速 率与底物浓度的双倒数曲线来确定. 如图 2 所示, 在不 同浓度的化合物 $\mathbf{C}_{\mathbf{8}}$ 和 $\mathbf{D}_{\mathbf{8}}$ 作用下, 酶促反应速率与底物 浓度的双倒数曲线在第二象限交于一点. 根据抑制剂类 型的定义可知, 化合物 $\mathbf{C}_{\mathbf{8}}$ 和 $\mathbf{D}_{\mathbf{8}}$ 抑制酪氨酸酶的抑制类 型是混合型抑制 ${ }^{[10]}$. 也就是说化合物 $\mathbf{C}_{\mathbf{8}}$ 和 $\mathbf{D}_{\mathbf{8}}$ 不仅可以 与游离酪氨酸酶结合, 还可与酪氨酸酶-底物复合物结 合. 抑制剂与游离酶结合的平衡常数 $\left(K_{\mathrm{I}}\right)$ 可通过不同浓
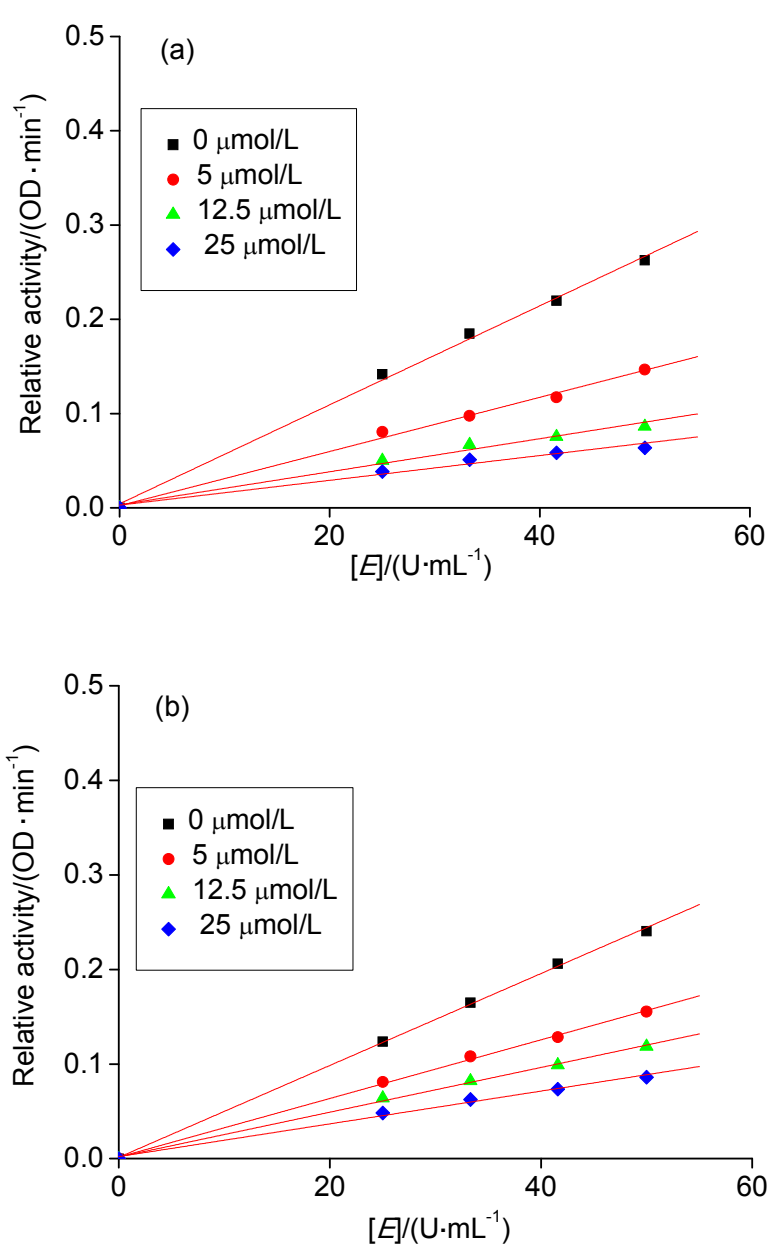

图 1 化合物 $\mathbf{C}_{\mathbf{8}}(\mathrm{a})$ 和 $\mathbf{D}_{\mathbf{8}}$ (b)抑制酪氨酸酶的机理 Figure 1 Inhibition mechanism of mushroom tyrosinase by $\mathbf{C}_{\mathbf{8}}$ (a) and $\mathbf{D}_{\mathbf{8}}$ (b)

度抑制剂双倒数曲线的斜率与浓度作图得出，抑制剂与 酶-底物复合物结合的平衡常数 $\left(K_{\mathrm{IS}}\right)$ 可通过不同浓度抑 制剂双倒数曲线的截距与浓度作图得出参考文献 ${ }^{[23]} . \mathbf{C}_{\mathbf{8}}$ 和 $\mathbf{D}_{\mathbf{8}}$ 的 $K_{\mathrm{I}}$ 分别为 $1.07,20.61 \mu \mathrm{mol} \cdot \mathrm{L}^{-1}, K_{\mathrm{IS}}$ 分别为 3.72 , $27.09 \mu \mathrm{mol} \cdot \mathrm{L}^{-1} . \mathbf{C}_{\mathbf{8}}$ 和 $\mathbf{D}_{\mathbf{8}}$ 的 $K_{\mathrm{IS}}$ 值高于 $K_{\mathrm{I}}$ 值, 表明 $\mathbf{C}_{\mathbf{8}}$ 和 $\mathbf{D}_{8}$ 更倾向与游离酶结合 ${ }^{[19]}$.

\section{4 分子对接}

用 Sybyl-2.1.1 软件模拟了化合物 $\mathbf{C}_{8}$ 和 $\mathbf{D}_{8}$ 与酪氨酸 酶结合情况, 酪氨酸酶晶体结构(PDB ID:2Y9X)从 PDB 数据库获得. 首先研究了化合物与酪氨酸酶活性口袋的 结合情况, 从图 $3 a \sim 3 b$ 可以看出, $\mathbf{C}_{\mathbf{8}}$ 和 $\mathbf{D}_{\mathbf{8}}$ 可以较好地 与酪氨酸酶活性口袋结合, 其中香豆素部分很好地嵌入 在活性口袋中，而肉桂酸部分则处于口袋的边缘. 接着 分析了化合物与酪氨酸酶活性口袋处氨基酸残基的结 合情况. 从图 $3 \mathrm{c}$ 可以看出化合物 $\mathbf{C}_{\mathbf{8}}$ 的羰基氧与酪氨酸 酶铜离子间存在极性键作用, $\mathbf{C}_{8}$ 羰基与 $\mathrm{Cu} 400$ 和 $\mathrm{Cu} 401$ 间距分别为 0.2264 和 $0.2256 \mathrm{~nm}$; 而且化合物 $\mathbf{C}_{\mathbf{8}}$ 与 


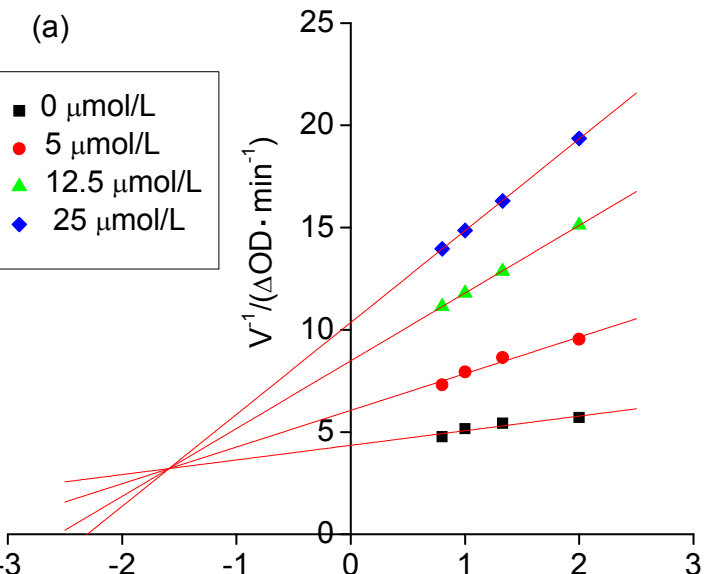

$[\mathrm{S}]^{-1} /\left(\mathrm{L} \cdot \mathrm{mmol}^{-1}\right)$

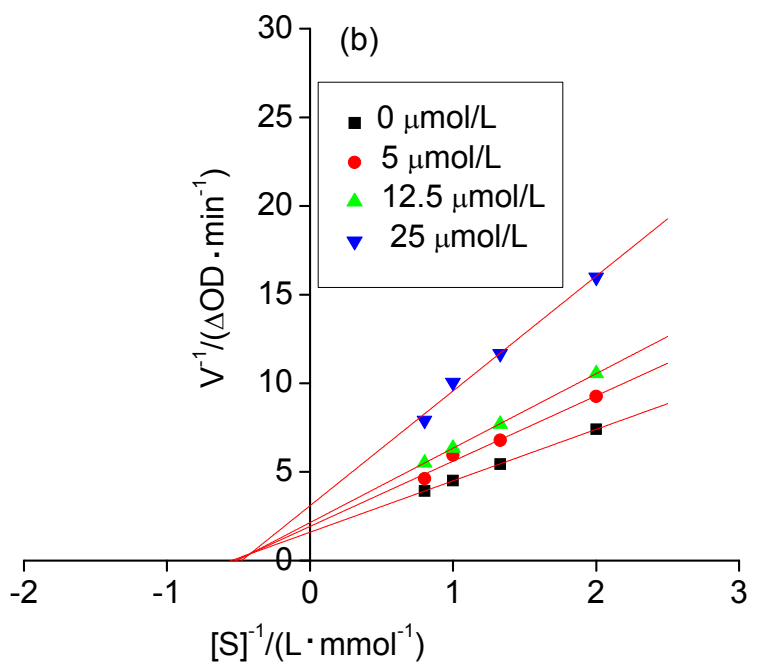

图 $2 C_{8}(a)$ 和 $D_{8}(b)$ 的双倒数曲线

Figure 2 Lineweaver-Burk plots of $\mathbf{C}_{\mathbf{8}}$ (a) and $\mathbf{D}_{\mathbf{8}}$ (b)
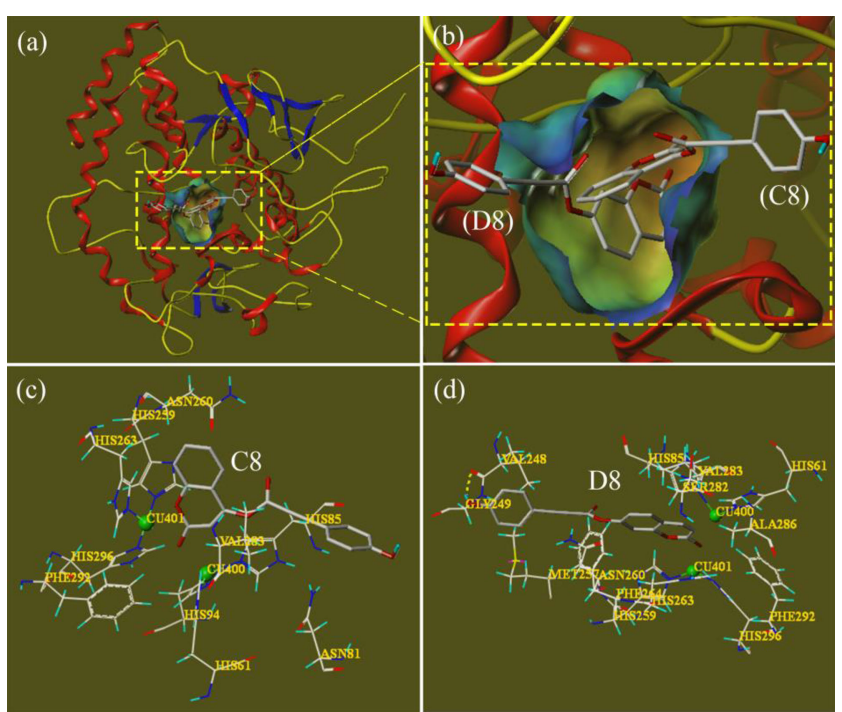

图 $3 \mathrm{C}_{8}$ 和 $\mathrm{D}_{8}$ 与酪氨酸酶的分子对接

Figure 3 Docking of $\mathbf{C}_{\mathbf{8}}$ and $\mathbf{D}_{\mathbf{8}}$ with tyrosinase (a, b) The docking of $\mathbf{C 8}$ and $\mathbf{D 8}$ with the pocket of tyrosinase; (c) the docking of $\mathbf{C 8}$ with amino acid residue of pocket of tyrosinase; (d) the docking of $\mathbf{D 8}$ with amino acid residue of pocket of tyrosinase
Asn81，Val283，Phe292，Asn260 间形成疏水作用. 化合 物 $\mathbf{D}_{\mathbf{8}}$ 的羰基氧同样与酪氨酸酶铜离子间形成极性键作 用, $\mathbf{D}_{\mathbf{8}}$ 羰基与 $\mathrm{Cu} 400$ 和 $\mathrm{Cu} 401$ 间距分别为 0.3601 和 $0.2047 \mathrm{~nm}$; 酚差基氧与 Val248 形成氢键(间距为 0.2751 $\mathrm{nm})$; 此外化合物 $\mathbf{D}_{\mathbf{8}}$ 还与 Gly249, Val283, Ser282, Ala286, Met257, Asn260, Phe264, Phe292 形成疏水作用 (图 3d). 相比于化合物 $\mathbf{C}_{8}$, 化合物 $\mathbf{D}_{8}$ 与酪氨酸酶的 Val248 形成了氢键, 这可能是两者抑制活性差异的重要 原因.

\section{2 结论}

合成了系列肉桂酸 - 香豆素酯类似物 $\left(\mathbf{C}_{1} \sim \mathrm{C}_{9}\right.$ 和 $\left.\mathbf{D}_{\mathbf{1}} \sim \mathbf{D}_{9}\right)$, 并篎选了他们的酪氨酸酶抑制活性. 肉桂酸 和香豆素骨架杂合后，酪氨酸酶抑制活性明显提高. 其 中化合物 $\mathbf{C}_{\mathbf{8}}\left(\mathrm{IC}_{50} 10.7 \mu \mathrm{mol} \cdot \mathrm{L}^{-1}\right)$ 和 $\mathbf{D}_{\mathbf{8}}\left(\mathrm{IC}_{50} 2.2 \mu \mathrm{mol} \cdot\right.$ $\left.\mathrm{L}^{-1}\right)$ 的酪氨酸酶抑制活性最强, 分别是曲酸 $\left(\mathrm{IC}_{50} 28.5\right.$ $\mu \mathrm{mol} \cdot \mathrm{L}^{-1}$ ) 的 3 倍和 13 倍. 动力学研究表明, 化合物 $\mathbf{C}_{8}$ 和 $\mathbf{D}_{\mathbf{8}}$ 是一种可逆混合型的酪氨酸酶抑制剂. 肉桂酸和 香豆素骨架杂合可开发为潜在的酪氨酸酶抑制剂.

\section{3 实验部分}

\section{1 仪器与试剂}

Bruker Advance DPX-500 MHz 核磁共振仪(以 TMS 为内标, Chloroform- $d$ 或 DMSO- $d_{6}$ 为溶剂)测定; 岛 津红外光谱仪 ( $\mathrm{KBr}$ 压片); Thermo Fisher Scientific(上海) $\left(\mathrm{LCQ}^{\mathrm{TM}}\right) \mathrm{LC}-\mathrm{MS}$ 质谱仪; 上海仪电物理光学仪器有限 公司 WRS-2C 熔点仪(温度计未经校正).

蘑菇酪氨酸酶 (EC 1.14.1.8.1) 和 $L$-多巴购自 Sigma-Aldrich; 7-羟基香豆素, 4-羟基香豆素, 曲酸购自 上海泰坦化学有限公司; 取代肉桂酸购自阿拉丁试剂 (上海)有限公司. 其他试剂均为市售分析纯.

\section{2 肉桂酸 - 香豆素酯类似物 $\left(C_{1} \sim C_{9}\right.$ 和 $\left.D_{1} \sim D_{9}\right)$ 的合 成}

将取代肉桂酸 $\mathbf{A}_{\mathbf{1}} \sim \mathbf{A}_{\mathbf{9}}(1.0 \mathrm{mmol}), \mathrm{SOCl}_{2}(2.2$ $\mathrm{mmol}$ ), $N, N$-二甲基甲酰胺(DMF) (1 滴)加入二氯甲烷 (DCM) $(5 \mathrm{~mL})$ 中, 反应 $5 \mathrm{~h}$, 浓缩可得对应的肉桂酰氯 $\mathbf{B}_{1} \sim \mathbf{B}_{9}$. 加入 $\mathrm{DCM}(5 \mathrm{~mL})$ 溶解后，4-羟基香豆素(1.2 $\mathrm{mmol})$ 或 7-羟基香豆素 $(1.2 \mathrm{mmol})$ 溶于 DCM $(5 \mathrm{~mL})$ 中, 在冰浴下缓慢滴加至肉桂酰氯溶液, 加入 $N, N$-二异丙基 乙胺 $(3.2 \mathrm{mmol}$ ), 薄层色谱(TLC)监测至反应完成. 用饱 和 $\mathrm{NaHCO}_{3}$ 淬灭反应，乙酸乙酯萃取后用饱和食盐水洗 涤, 无水硫酸钠干燥后浓缩, 柱层析分离纯化可得到 $\mathrm{C}_{\mathbf{1}} \sim \mathrm{C}_{\mathbf{9}}$ 和 $\mathrm{D}_{\mathbf{1}} \sim \mathrm{D}_{\mathbf{9}}$.

2 -氧代- $2 H$-苯并吡喃-4-基肉桂酸酯 $\left(\mathbf{C}_{1}\right)$ : 淋洗剂: 石油醚/乙酸乙酯/二氯甲烷 $(V: V: V=5: 1: 1,500$ 
$\mathrm{mL}$ ). 白色固体, 产率 $66.3 \%$. m.p. $154 \sim 157{ }^{\circ} \mathrm{C}$ (Lit. ${ }^{[32]}$ m.p. $\left.156 \sim 157{ }^{\circ} \mathrm{C}\right) ;{ }^{1} \mathrm{H}$ NMR $\left(500 \mathrm{MHz}, \mathrm{CDCl}_{3}\right) \delta: 7.98$ $(\mathrm{d}, J=16.0 \mathrm{~Hz}, 1 \mathrm{H}, \mathrm{CH}=\mathrm{CH}), 7.71(\mathrm{dd}, J=7.9,1.6 \mathrm{~Hz}$, $1 \mathrm{H}, \mathrm{C} 21-\mathrm{ArH}), 7.66 \sim 7.57(\mathrm{~m}, 3 \mathrm{H}, \mathrm{C} 4, \mathrm{C} 6, \mathrm{C} 2-\mathrm{ArH})$, $7.51 \sim 7.44$ (m, 3H, C1, C3, C19-ArH), 7.38 (dd, $J=8.4$, $1.1 \mathrm{~Hz}, 1 \mathrm{H}, \mathrm{C} 22-\mathrm{ArH}), 7.35 \sim 7.30(\mathrm{~m}, 1 \mathrm{H}, \mathrm{C} 20-\mathrm{ArH})$, $6.68(\mathrm{~d}, J=16.0 \mathrm{~Hz}, 1 \mathrm{H}, \mathrm{CH}=\mathrm{CH}), 6.60(\mathrm{~s}, 1 \mathrm{H}, \mathrm{CH}=\mathrm{C})$; ${ }^{13} \mathrm{C} \mathrm{NMR}\left(126 \mathrm{MHz}, \mathrm{CDCl}_{3}\right) \delta: 162.8,161.6,158.7,153.7$, $149.4,133.5,132.8,131.6,129.2,128.7,124.4,122.9$, 117.1, 115.7, 115.4, 105.2; IR (KBr) v: 2924, 1706, 1612, $1385,1328,1116,940,841,736 \mathrm{~cm}^{-1}$; MS (70 eV) $\mathrm{m} / \mathrm{z}$ : $315.57\left([\mathrm{M}+\mathrm{Na}]^{+}\right)$.

2-氧代- $2 H$-苯并吡喃-4-基( $E$ )-3-(对甲苯基)丙烯酸 酯 $\left(\mathbf{C}_{2}\right)$ : 淋洗剂: 石油醚/乙酸乙酯/二氯甲烷 $(V: V:$ $V=5: 1: 1(400 \mathrm{~mL})$. 白色固体, 产率 53.5\%. m.p. 138 $\sim 140{ }^{\circ} \mathrm{C}$ (Lit. ${ }^{[32]}$ m.p. $139 \sim 140{ }^{\circ} \mathrm{C}$ ); ${ }^{1} \mathrm{H}$ NMR $(500$ $\left.\mathrm{MHz}, \mathrm{CDCl}_{3}\right) \delta: 7.95(\mathrm{~d}, J=15.9 \mathrm{~Hz}, 1 \mathrm{H}, \mathrm{CH}=\mathrm{CH}), 7.72$ (dd, $J=8.0,1.6 \mathrm{~Hz}, 1 \mathrm{H}, \mathrm{C} 19-\mathrm{ArH}), 7.59(\mathrm{td}, J=8.3,1.6$ $\mathrm{Hz}, 1 \mathrm{H}, \mathrm{C} 21-\mathrm{ArH}), 7.53$ (d, $J=8.0 \mathrm{~Hz}, 2 \mathrm{H}, \mathrm{C} 4, \mathrm{C} 6-\mathrm{ArH}$ ), $7.38(\mathrm{~d}, J=8.3 \mathrm{~Hz}, 1 \mathrm{H}, \mathrm{C} 22-\mathrm{ArH}), 7.32(\mathrm{t}, J=7.6 \mathrm{~Hz}, 1 \mathrm{H}$, C20-ArH), $7.29 \sim 7.25$ (m, 2H, C1,C3-ArH), 6.63 (d, $J=$ $16.0 \mathrm{~Hz}, 1 \mathrm{H}, \mathrm{CH}=\mathrm{CH}), 6.60(\mathrm{~s}, 1 \mathrm{H}, \mathrm{CH}=\mathrm{C}), 2.42(\mathrm{~s}, 3 \mathrm{H}$, $\left.\mathrm{CH}_{3}\right) ;{ }^{13} \mathrm{C}$ NMR $\left(126 \mathrm{MHz}, \mathrm{CDCl}_{3}\right) \delta: 163.0,161.6,158.7$, $154.1,153.7,149.4,142.4,132.7,130.9,130.0,128.7$, 124.3, 122.9, 117.1, 115.7, 114.1, 105.2, 21.7; IR (KBr) v: 2923, 1713, 1616, 1385, 1326, 1127, 938, 846, 810, 759 $\mathrm{cm}^{-1}$; MS (70 eV) $m / z: 329.07\left([\mathrm{M}+\mathrm{Na}]^{+}\right)$.

2-氧代- $2 \mathrm{H}$-苯并吡喃-4-基( $E$ )-3-(4-甲氧基苯基)丙 烯酸酯 $\left(\mathbf{C}_{3}\right)$ : 淋洗剂: 石油醚/乙酸乙酯/二氯甲烷 $(V$ : $V: V=5: 1: 1(350 \mathrm{~mL})$. 白色固体, 产率 54.5\%. m.p. $150 \sim 152{ }^{\circ} \mathrm{C}$ (Lit. ${ }^{[32]}$ m.p. $\left.149 \sim 151{ }^{\circ} \mathrm{C}\right) ;{ }^{1} \mathrm{H}$ NMR $(500$ $\left.\mathrm{MHz}, \mathrm{CDCl}_{3}\right) \delta: 7.93(\mathrm{~d}, J=15.8 \mathrm{~Hz}, 1 \mathrm{H}, \mathrm{CH}=\mathrm{CH}), 7.72$ (dd, $J=7.9,1.6 \mathrm{~Hz}, 1 \mathrm{H}, \mathrm{C} 21-\mathrm{ArH}), 7.62 \sim 7.57(\mathrm{~m}, 3 \mathrm{H}$, C4, C6, C19-ArH), 7.38 (dd, $J=8.4,1.1 \mathrm{~Hz}, 1 \mathrm{H}, \mathrm{C} 22-$ ArH), 7.32 (td, $J=7.7,1.2 \mathrm{~Hz}, 1 \mathrm{H}, \mathrm{C} 20-\mathrm{ArH}), 7.00 \sim 6.95$ (m, 2H, C1, C3-ArH), 6.59 (s, 1H, CH=C), $6.54(\mathrm{~d}, J=$ $15.8 \mathrm{~Hz}, 1 \mathrm{H}, \mathrm{CH}=\mathrm{CH}), 3.88\left(\mathrm{~s}, 3 \mathrm{H}, \mathrm{OCH}_{3}\right) ;{ }^{13} \mathrm{C} \mathrm{NMR}$ $\left(126 \mathrm{MHz}, \mathrm{CDCl}_{3}\right) \delta: 163.1,162.5,161.7,158.8,153.7$, $149.1,132.7,130.6,126.3,124.3,123.0,117.1,115.8$, 114.7, 112.5, 105.1, 55.5; IR (KBr) v: 2925, 1728, 1609, 1381, 1293, 1112, 946, 837, $765 \mathrm{~cm}^{-1}$; MS (70 eV) $\mathrm{m} / \mathrm{z}$ : $344.98\left([\mathrm{M}+\mathrm{Na}]^{+}\right)$.

2-氧代- $2 H$-苯并吡喃-4-基( $E$ )-3-(4-氟苯基)丙烯酸 酯 $\left(\mathbf{C}_{4}\right)$ : 淋洗剂: 石油醚/乙酸乙酯/二氯甲烷 $(V: V: V$ $=5: 1: 1(400 \mathrm{~mL})$. 白色固体, 产率 54.5\%. m.p. 146
$149{ }^{\circ} \mathrm{C}$ (Lit. ${ }^{[32]}$ m.p. $\left.146 \sim 147{ }^{\circ} \mathrm{C}\right) ;{ }^{1} \mathrm{H}$ NMR $(500 \mathrm{MHz}$, $\left.\mathrm{CDCl}_{3}\right) \delta: 7.93(\mathrm{~d}, J=15.9 \mathrm{~Hz}, 1 \mathrm{H}, \mathrm{CH}=\mathrm{CH}), 7.70(\mathrm{dd}$, $J=8.0,1.6 \mathrm{~Hz}, 1 \mathrm{H}, \mathrm{C} 19-\mathrm{ArH}), 7.66 \sim 7.57(\mathrm{~m}, 3 \mathrm{H}, \mathrm{C} 4$, C6, C21-ArH), 7.38 (dd, $J=8.4,1.1 \mathrm{~Hz}, 1 \mathrm{H}, \mathrm{C} 20-\mathrm{ArH})$, $7.34 \sim 7.29(\mathrm{~m}, 1 \mathrm{H}, \mathrm{C} 22-\mathrm{ArH}), 7.18 \sim 7.13(\mathrm{~m}, 2 \mathrm{H}$, $\mathrm{C} 1, \mathrm{C} 3-\mathrm{ArH}), 6.63 \sim 6.58(\mathrm{~m}, 2 \mathrm{H}, \mathrm{CH}=\mathrm{CH}, \mathrm{CH}=\mathrm{C}) ;{ }^{13} \mathrm{C}$ NMR $\left(126 \mathrm{MHz}, \mathrm{CDCl}_{3}\right) \delta: 165.7,163.7,162.7,161.6$, $158.6,153.7,148.0,132.8,130.8,130.7,129.9,129.8$, $124.4,122.9,117.2,116.6,116.4,115.6,115.2,115.1$, 105.3; IR (KBr) v: 2923, 1711, 1619, 1387, 1331, 1133, 939, 837, $758 \mathrm{~cm}^{-1}$; MS $(70 \mathrm{eV}) \mathrm{m} / \mathrm{z}: 333.39\left([\mathrm{M}+\mathrm{Na}]^{+}\right)$.

2-氧代- $2 H$-苯并吡喃-4-基 $(E)$-3-(4-氯苯基)丙烯酸 酯 $\left(\mathbf{C}_{5}\right)$ : 淋洗剂: 石油醚/乙酸乙酯/二氯甲烷 $(V: V: V$ $=5: 1: 1(400 \mathrm{~mL})$. 白色固体, 产率 62.3\%. m.p. 184 $186{ }^{\circ} \mathrm{C}$ (Lit. ${ }^{[32]}$ m.p. $184 \sim 185{ }^{\circ} \mathrm{C}$ ); ${ }^{1} \mathrm{H}$ NMR $(500 \mathrm{MHz}$, $\left.\mathrm{CDCl}_{3}\right) \delta: 7.92(\mathrm{~d}, J=15.9 \mathrm{~Hz}, 1 \mathrm{H}, \mathrm{CH}=\mathrm{CH}), 7.70(\mathrm{dd}$, $J=7.9,1.6 \mathrm{~Hz}, 1 \mathrm{H}, \mathrm{C} 21-\mathrm{ArH}), 7.62 \sim 7.55(\mathrm{~m}, 3 \mathrm{H}, \mathrm{C} 1$, C3, C19-ArH), 7.46 7.42 (m, 2H, C4, C6-ArH), 7.39 (dd, $J=8.4,1.1 \mathrm{~Hz}, 1 \mathrm{H}, \mathrm{C} 22-\mathrm{ArH}), 7.32$ (ddd, $J=8.2,7.3,1.1$ $\mathrm{Hz}, 1 \mathrm{H}, \mathrm{C} 20-\mathrm{ArH}), 6.66(\mathrm{~d}, J=16.0 \mathrm{~Hz}, 1 \mathrm{H}, \mathrm{CH}=\mathrm{CH})$, $6.59(\mathrm{~s}, 1 \mathrm{H}, \mathrm{CH}=\mathrm{C}) ;{ }^{13} \mathrm{C}$ NMR $\left(126 \mathrm{MHz}, \mathrm{CDCl}_{3}\right) \delta$ : $162.6,161.5,158.6,153.7,147.8,137.7,132.8,132.0$, $129.8,129.6,124.4,122.8,117.2,115.9,115.6,105.3$; IR (KBr) $v$ : 2922, 1625, 1382, 1317, 1131, 940, 868, 821, 755 $\mathrm{cm}^{-1}$; MS (70 eV) $m / z: 348.84\left([\mathrm{M}+\mathrm{Na}]^{+}\right)$.

2-氧代- $2 H$-苯并吡喃-4-基 $(E)$-3-(4-溴苯基)丙烯酸 酯 $\left(\mathbf{C}_{6}\right)$ : 淋洗剂: 石油醚/乙酸乙酯/二氯甲烷 $(V: V: V$ $=5: 1: 1(400 \mathrm{~mL})$. 白色固体, 产率 61.7\%. m.p. 194 $197{ }^{\circ} \mathrm{C}$ (Lit. ${ }^{[32]}$ m.p. $194 \sim 195{ }^{\circ} \mathrm{C}$ ); ${ }^{1} \mathrm{H}$ NMR $(500 \mathrm{MHz}$, $\left.\mathrm{CDCl}_{3}\right) \delta: 7.91(\mathrm{~d}, J=16.0 \mathrm{~Hz}, 1 \mathrm{H}, \mathrm{CH}=\mathrm{CH}), 7.70(\mathrm{dd}$, $J=7.9,1.6 \mathrm{~Hz}, 1 \mathrm{H}, \mathrm{C} 19-\mathrm{ArH}), 7.65 \sim 7.57$ (m, 3H, C4, C6, C21-ArH), 7.54 7.47 (m, 2H, C1, C3-ArH), 7.39 (dd, $J=8.4,1.1 \mathrm{~Hz}, 1 \mathrm{H}, \mathrm{C} 22-\mathrm{ArH}), 7.35 \sim 7.28(\mathrm{~m}, 1 \mathrm{H}$, C20-ArH), $6.68(\mathrm{~d}, J=16.0 \mathrm{~Hz}, 1 \mathrm{H}, \mathrm{CH}=\mathrm{CH}), 6.59(\mathrm{~s}$, $1 \mathrm{H}, \mathrm{CH}=\mathrm{C}) ;{ }^{13} \mathrm{C}$ NMR $\left(126 \mathrm{MHz}, \mathrm{CDCl}_{3}\right) \delta: 162.6$, $161.5,158.6,153.7,147.9,132.8,132.5,132.4,132.3$, 130.0, 126.1, 124.4, 122.8, 117.2, 116.0, 115.6, 105.3; IR (KBr) v: 2922, 1755, 1623, 1380, 1314, 1128, 938, 867, $818,754 \mathrm{~cm}^{-1}$; MS $(70 \mathrm{eV}) \mathrm{m} / z: 393.05\left([\mathrm{M}+\mathrm{Na}]^{+}\right)$.

2-氧代- $2 H$-苯并吡喃-4-基 (E)-3-(4-三氟甲基苯基) 丙烯酸酯 $\left(\mathbf{C}_{7}\right)$ : 淋洗剂: 石油醚/乙酸乙酯/二氯甲烷 $(V$ : $V: V=5: 1: 1(350 \mathrm{~mL})$. 白色固体, 产率 58.9\%. m.p. $164 \sim 166{ }^{\circ} \mathrm{C}$ (Lit. ${ }^{[32]}$ m.p. $165 \sim 166{ }^{\circ} \mathrm{C}$ ); ${ }^{1} \mathrm{H}$ NMR $(500$ $\left.\mathrm{MHz}, \mathrm{CDCl}_{3}\right) \delta: 7.99(\mathrm{~d}, J=16.0 \mathrm{~Hz}, 1 \mathrm{H}, \mathrm{CH}=\mathrm{CH})$, $7.81 \sim 7.67$ (m, 5H, C1, C3, C4, C6, C19-ArH), 7.61 (ddd, 
$J=8.8,7.3,1.6 \mathrm{~Hz}, 1 \mathrm{H}, \mathrm{C} 20-\mathrm{ArH}), 7.39$ (dd, $J=8.4,1.1$ $\mathrm{Hz}, 1 \mathrm{H}, \mathrm{C} 22-\mathrm{ArH}), 7.36 \sim 7.30$ (m, 1H, C21-ArH), 6.77 (d, $J=16.0 \mathrm{~Hz}, 1 \mathrm{H}, \mathrm{CH}=\mathrm{CH}), 6.60(\mathrm{~s}, 1 \mathrm{H}, \mathrm{CH}=\mathrm{C}) ;{ }^{13} \mathrm{C}$ NMR $\left(126 \mathrm{MHz}, \mathrm{CDCl}_{3}\right) \delta: 162.2,161.4,158.5,153.7$, $147.3,136.8,133.0,132.9,132.8,128.8,126.2,126.1$, 124.4, 122.8, 118.0, 117.2, 115.5, 105.4; IR (KBr) v: 2924, $1754,1626,1383,1323,1117,941,834,755 \mathrm{~cm}^{-1}$; MS (70 eV) $m / z: 382.78\left([\mathrm{M}+\mathrm{Na}]^{+}\right)$.

2-氧代- $2 H$-苯并吡喃-4-基( $E$ )-3-(4-羟基苯基)丙烯 酸酯 $\left(\mathbf{C}_{8}\right)$ : 淋洗剂: 石油醚/乙酸乙酯/二氯甲烷 $(V: V$ : $V=5: 1: 1(600 \mathrm{~mL})$. 白色固体, 产率 49.7\%. m.p. $170 \sim 173{ }^{\circ} \mathrm{C}$ (Lit. ${ }^{[32]}$ m.p. $\left.170 \sim 171{ }^{\circ} \mathrm{C}\right) ;{ }^{1} \mathrm{H}$ NMR $(500$ $\left.\mathrm{MHz}, \mathrm{CDCl}_{3}\right) \delta: 10.26(\mathrm{~s}, 1 \mathrm{H}, \mathrm{OH}), 7.91(\mathrm{~d}, J=15.9 \mathrm{~Hz}$, $1 \mathrm{H}, \mathrm{CH}=\mathrm{CH}), 7.79(\mathrm{dd}, J=7.9,1.6 \mathrm{~Hz}, 1 \mathrm{H}, \mathrm{C} 21-\mathrm{ArH})$, $7.74 \sim 7.67$ (m, 3H, C4, C6, C19-ArH), 7.49 (dd, $J=8.3$, $1.0 \mathrm{~Hz}, 1 \mathrm{H}, \mathrm{C} 22-\mathrm{ArH}), 7.40$ (td, $J=7.7,1.1 \mathrm{~Hz}, 1 \mathrm{H}$, C20-ArH), $6.87 \sim 6.82(\mathrm{~m}, 2 \mathrm{H}, \mathrm{C} 1, \mathrm{C} 3-\mathrm{ArH}), 6.77$ (d, $J=$ $15.9 \mathrm{~Hz}, 1 \mathrm{H}, \mathrm{CH}=\mathrm{CH}), 6.62(\mathrm{~s}, 1 \mathrm{H}, \mathrm{CH}=\mathrm{C}) ;{ }^{13} \mathrm{C} \mathrm{NMR}$ (126 MHz, DMSO) $\delta: 163.8,161.4,161.2,159.3,153.6$, $149.6,133.7,131.8,130.6,125.3,125.2,124.4,123.7$, 123.6, 117.2, 116.9, 116.4, 116.2, 112.0, 105.6; IR (KBr) $v$ : $3435,2925,1737,1596,1511,1384,1146,894,847,755$ $\mathrm{cm}^{-1}$; MS (70 eV) $m / z: 330.72\left([\mathrm{M}+\mathrm{Na}]^{+}\right)$.

2-氧代- $2 H$-苯并吡喃-4-基 $(E)$-3-(4-羟基-3-甲氧基苯 基)丙烯酸酯 $\left(\mathbf{C}_{9}\right)$ : 淋洗剂: 石油醚/乙酸乙酯/二氯甲烷 $(V: V: V=5: 1: 1(550 \mathrm{~mL})$. 淡黄色固体, 产率 50.0\%. m.p. $177 \sim 179{ }^{\circ} \mathrm{C}$ (Lit. ${ }^{[32]}$ m.p. $177 \sim 178{ }^{\circ} \mathrm{C}$ ); ${ }^{1} \mathrm{H}$ NMR $\left(500 \mathrm{MHz}\right.$, DMSO- $\left.d_{6}\right) \delta$ : $9.86(\mathrm{~s}, 1 \mathrm{H}, \mathrm{OH}), 7.90(\mathrm{~d}$, $J=15.8 \mathrm{~Hz}, 1 \mathrm{H}, \mathrm{CH}=\mathrm{CH}), 7.80(\mathrm{dd}, J=8.0,1.6 \mathrm{~Hz}, 1 \mathrm{H}$, C19-ArH), 7.72 (ddd, $J=8.7,7.3,1.6 \mathrm{~Hz}, 1 \mathrm{H}, \mathrm{C} 21-\mathrm{ArH}$ ), $7.52 \sim 7.46(\mathrm{~m}, 2 \mathrm{H}, \mathrm{C} 4, \mathrm{C} 22-\mathrm{ArH}), 7.45 \sim 7.40(\mathrm{~m}, 1 \mathrm{H}$, $\mathrm{C} 20-\mathrm{ArH}), 7.31 \sim 7.24(\mathrm{~m}, 1 \mathrm{H}, \mathrm{C} 6-\mathrm{ArH}), 6.88 \sim 6.82(\mathrm{~m}$, $2 \mathrm{H}, \mathrm{CH}=\mathrm{CH}, \mathrm{C} 1-\mathrm{ArH}), 6.63(\mathrm{~s}, 1 \mathrm{H}, \mathrm{CH}=\mathrm{C}), 3.85(\mathrm{~s}, 3 \mathrm{H}$, $\left.\mathrm{OCH}_{3}\right) ;{ }^{13} \mathrm{C}$ NMR (126 MHz, DMSO) $\delta$ : 163.8, 161.2, $159.3,153.6,151.0,149.9,148.6,133.7,125.7,125.2$, $125.0,123.7,117.2,116.1,115.9,112.3,112.2,105.6$, 56.3; IR (KBr) v: 3431, 2925, 1723, 1621, 1385, 1274, $1117,944,851,759 \mathrm{~cm}^{-1}$; MS (70 eV) m/z: $359.46([\mathrm{M}+$ $\mathrm{Na}]^{+}$).

2-氧代- $2 H$-苯并吡喃-7-基肉桂酸酯 $\left(\mathbf{D}_{1}\right)$ : 淋洗剂: 石油醚/乙酸乙酯/二氯甲烷 $(V: V: V=5: 1: 1(350$ $\mathrm{mL}$ ). 白色固体, 产率 $65.4 \%$. m.p. $153 \sim 154{ }^{\circ} \mathrm{C}$ (Lit. ${ }^{[32]}$ m.p. $\left.152 \sim 154{ }^{\circ} \mathrm{C}\right) ;{ }^{1} \mathrm{H}$ NMR $\left(500 \mathrm{MHz}, \mathrm{CDCl}_{3}\right) \delta: 7.91$ $(\mathrm{d}, J=16.0 \mathrm{~Hz}, 1 \mathrm{H}, \mathrm{CH}=\mathrm{CH}), 7.71(\mathrm{~d}, J=9.5 \mathrm{~Hz}, 1 \mathrm{H}$, $\mathrm{CH}=\mathrm{C}), 7.63 \sim 7.57(\mathrm{~m}, 2 \mathrm{H}, \mathrm{C} 1, \mathrm{C} 3-\mathrm{ArH}), 7.52(\mathrm{~d}, J=8.4$
Hz, 1H, C17-ArH), 7.48 7.41 (m, 3H, C16, C4, C6-ArH), 7.21 (d, $J=2.2 \mathrm{~Hz}, 1 \mathrm{H}, \mathrm{C} 13-\mathrm{ArH}), 7.15$ (dd, $J=8.4,2.2$ $\mathrm{Hz}, 1 \mathrm{H}, \mathrm{C} 2-\mathrm{ArH}), 6.63(\mathrm{~d}, J=16.0 \mathrm{~Hz}, 1 \mathrm{H}, \mathrm{CH}=\mathrm{CH})$, 6.41 (d, $J=9.6 \mathrm{~Hz}, 1 \mathrm{H}, \mathrm{C} 20-\mathrm{ArH}) ;{ }^{13} \mathrm{C}$ NMR $(126 \mathrm{MHz}$, $\left.\mathrm{CDCl}_{3}\right) \delta: 164.7,160.4,154.8,153.4,147.8,142.9,133.9$, $131.1,129.1,128.6,128.5,118.5,116.7,116.4,116.1$, 110.5; IR (KBr) v: 2925, 1725, 1623, 1410, 1312, 986, 864, 757, $692 \mathrm{~cm}^{-1}$; MS $(70 \mathrm{eV}) \mathrm{m} / z: 314.96\left([\mathrm{M}+\mathrm{Na}]^{+}\right)$.

2-氧代- $2 \mathrm{H}$-苯并吡喃-7-基( $E$ )-3-(对甲苯基)丙烯酸 酯 $\left(\mathbf{D}_{2}\right)$ : 淋洗剂: 石油醚/乙酸乙酯/二氯甲烷 $(V: V:$ $V=5: 1: 1(400 \mathrm{~mL})$. 白色固体, 产率 53.7\%. m.p. 174 $179{ }^{\circ} \mathrm{C}$ (Lit. ${ }^{[32]}$ m.p. $\left.174 \sim 176{ }^{\circ} \mathrm{C}\right) ;{ }^{1} \mathrm{H}$ NMR $(500$ $\left.\mathrm{MHz}, \mathrm{CDCl}_{3}\right) \delta: 7.88(\mathrm{~d}, J=16.0 \mathrm{~Hz}, 1 \mathrm{H}, \mathrm{CH}=\mathrm{CH}), 7.71$ $(\mathrm{d}, J=9.5 \mathrm{~Hz}, 1 \mathrm{H}, \mathrm{CH}=\mathrm{C}), 7.51(\mathrm{t}, J=8.0 \mathrm{~Hz}, 3 \mathrm{H}, \mathrm{C} 4$, C6, C16-ArH), 7.28 7.22 (m, 3H, C1, C3, C13-ArH), 7.21 (d, $J=2.2 \mathrm{~Hz}, 1 \mathrm{H}, \mathrm{C} 17-\mathrm{ArH}), 7.15$ (dd, $J=8.4,2.2$ $\mathrm{Hz}, 1 \mathrm{H}, \mathrm{CH}=\mathrm{CH}), 6.41(\mathrm{~d}, J=9.5 \mathrm{~Hz}, 1 \mathrm{H}, \mathrm{C} 20-\mathrm{ArH})$, $2.41\left(\mathrm{~s}, 3 \mathrm{H}, \mathrm{CH}_{3}\right) ;{ }^{13} \mathrm{C} \mathrm{NMR}\left(126 \mathrm{MHz}, \mathrm{CDCl}_{3}\right) \delta: 164.9$, $160.5,154.8,153.5,147.8,143.0,141.7,131.2,129.9$, $128.6,128.5,118.6,116.6,116.0,115.3,110.5,21.6$; IR (KBr) $v: 2923,1723,1619,1404,1260,1129,987,811$, $747 \mathrm{~cm}^{-1}$; MS $(70 \mathrm{eV}) \mathrm{m} / z: 328.97\left([\mathrm{M}+\mathrm{Na}]^{+}\right)$.

2-氧代- $2 H$-苯并吡喃-7-基 $(E)$-3-(4-甲氧基苯基)丙 烯酸酯 $\left(\mathbf{D}_{3}\right)$ : 淋洗剂: 石油醚/乙酸乙酯/二氯甲烷 $(V$ : $V: V=5: 1: 1(350 \mathrm{~mL})$. 白色固体, 产率 53.7\%. m.p. 196 $200{ }^{\circ} \mathrm{C}$ (Lit. ${ }^{[32]}$ m.p. $\left.196 \sim 198{ }^{\circ} \mathrm{C}\right) ;{ }^{1} \mathrm{H}$ NMR (500 $\left.\mathrm{MHz}, \mathrm{CDCl}_{3}\right) \delta: 7.86(\mathrm{~d}, J=15.9 \mathrm{~Hz}, 1 \mathrm{H}, \mathrm{CH}=\mathrm{CH}), 7.70$ $(\mathrm{d}, J=9.5 \mathrm{~Hz}, 1 \mathrm{H}, \mathrm{CH}=\mathrm{C}), 7.58 \sim 7.49(\mathrm{~m}, 3 \mathrm{H}, \mathrm{C} 4, \mathrm{C} 6$, C16-ArH), 7.22 7.12 (m, 2H, C13, C17-ArH), 6.95 (d, $J=2.0 \mathrm{~Hz}, 2 \mathrm{H}, \mathrm{C} 1-\mathrm{ArH}), 6.94(\mathrm{~d}, J=2.1 \mathrm{~Hz}, 1 \mathrm{H}$, C3-ArH), $6.49(\mathrm{~d}, J=15.9 \mathrm{~Hz}, 1 \mathrm{H}, \mathrm{CH}=\mathrm{CH}), 6.40(\mathrm{~d}, J=$ $9.5 \mathrm{~Hz}, 1 \mathrm{H}, \mathrm{C} 20-\mathrm{ArH}), 3.86$ (s, 3H, $\left.\mathrm{OCH}_{3}\right) ;{ }^{13} \mathrm{C} \mathrm{NMR}(126$ $\left.\mathrm{MHz}, \mathrm{CDCl}_{3}\right) \delta: 165.0,162.1,160.5,154.8,153.6,147.4$, $143.0,130.3,128.6,126.7,118.6,116.6,116.0,114.6$, 113.7, 110.6, 55.5; IR (KBr) v: 2925, 1736, 1610, 1417, 1260, 1136, 995, 887, $825 \mathrm{~cm}^{-1}$; MS (70 eV) m/z: 345.10 $\left([\mathrm{M}+\mathrm{Na}]^{+}\right)$.

2-氧代- $2 H$-苯并吡喃-7-基 $(E)$-3-(4-氟苯基)丙烯酸 酯 $\left(\mathbf{D}_{4}\right)$ : 淋洗剂: 石油醚/乙酸乙酯/二氯甲烷 $(V: V:$ $V=5: 1: 1(350 \mathrm{~mL})$. 白色固体, 产率 63.8\%. m.p. $207 \sim 210{ }^{\circ} \mathrm{C}$ (Lit. ${ }^{[32]}$ m.p. $208 \sim 210{ }^{\circ} \mathrm{C}$ ); ${ }^{1} \mathrm{H}$ NMR $(500$ $\left.\mathrm{MHz}, \mathrm{CDCl}_{3}\right) \delta: 7.87(\mathrm{~d}, J=16.0 \mathrm{~Hz}, 1 \mathrm{H}, \mathrm{CH}=\mathrm{CH}), 7.71$ $(\mathrm{d}, J=9.6 \mathrm{~Hz}, 1 \mathrm{H}, \mathrm{CH}=\mathrm{C}), 7.64 \sim 7.57(\mathrm{~m}, 2 \mathrm{H}, \mathrm{C} 4$, C6-ArH), 7.52 (d, $J=8.4 \mathrm{~Hz}, 1 \mathrm{H}, \mathrm{C} 16-\operatorname{ArH}), 7.21$ (d, $J=$ $2.2 \mathrm{~Hz}, 1 \mathrm{H}, \mathrm{C} 17-\mathrm{ArH}), 7.13$ (td, $J=8.4,2.0 \mathrm{~Hz}, 3 \mathrm{H}, \mathrm{C} 1$, 
C3, C13-ArH), $6.56(\mathrm{~d}, J=15.9 \mathrm{~Hz}, 1 \mathrm{H}, \mathrm{CH}=\mathrm{CH}), 6.41$ (d, $J=9.6 \mathrm{~Hz}, 1 \mathrm{H}, \mathrm{C} 20-\mathrm{ArH}) ;{ }^{13} \mathrm{C}$ NMR $(126 \mathrm{MHz}$, $\left.\mathrm{CDCl}_{3}\right) \delta: 164.6,160.4,154.8,153.4,146.4,142.9,130.5$, $130.4,128.6,118.5,116.4,116.3,116.2,116.2,116.1$, 110.5; IR (KBr) v: 2924, 1734, 1615, 1411, 1235, 1137, 993, $835 \mathrm{~cm}^{-1}$; MS $(70 \mathrm{eV}) \mathrm{m} / \mathrm{z}: 333.11\left([\mathrm{M}+\mathrm{Na}]^{+}\right)$.

2-氧代- $2 H$-苯并吡喃-7-基( $E$ )-3-(4-氯苯基) 丙烯酸 酯(D5): 淋洗剂: 石油醚/乙酸乙酯/二氯甲烷 $(V: V$ : $V=5: 1: 1(400 \mathrm{~mL})$. 白色固体, 产率 51.7\%. m.p. 200 203 ${ }^{\circ} \mathrm{C}$ (Lit. ${ }^{[32]}$ m.p. $\left.201 \sim 203{ }^{\circ} \mathrm{C}\right) ;{ }^{1} \mathrm{H}$ NMR $(500$ $\left.\mathrm{MHz}, \mathrm{CDCl}_{3}\right) \delta: 7.85(\mathrm{~d}, J=16.0 \mathrm{~Hz}, 1 \mathrm{H}, \mathrm{CH}=\mathrm{CH}), 7.71$ $(\mathrm{d}, J=9.5 \mathrm{~Hz}, 1 \mathrm{H}, \mathrm{CH}=\mathrm{C}), 7.57 \sim 7.50(\mathrm{~m}, 3 \mathrm{H}, \mathrm{C} 4, \mathrm{C} 6$, C16-ArH), $7.45 \sim 7.38$ (m, 2H, C1, C3-ArH), 7.20 (d, $J=$ $2.2 \mathrm{~Hz}, 1 \mathrm{H}, \mathrm{C} 13-\mathrm{ArH}), 7.14(\mathrm{dd}, J=8.5,2.2 \mathrm{~Hz}, 1 \mathrm{H}$, C17-ArH), $6.60(\mathrm{~d}, J=16.0 \mathrm{~Hz}, 1 \mathrm{H}, \mathrm{CH}=\mathrm{CH}), 6.41(\mathrm{~d}$, $J=9.5 \mathrm{~Hz}, 1 \mathrm{H}, \mathrm{C} 20-\mathrm{ArH}) ;{ }^{13} \mathrm{C} \mathrm{NMR}\left(126 \mathrm{MHz}, \mathrm{CDCl}_{3}\right) \delta$ : $164.5,160.4,154.8,153.3,146.2,142.9,137.1,132.4$, 129.6, 129.4, 128.6, 118.4, 117.0, 116.7, 116.1, 110.5; IR (KBr) v: 2924, 1726, 1620, 1406, 1273, 1125, $817 \mathrm{~cm}^{-1}$; MS (70 eV) $m / z: 349.31\left([\mathrm{M}+\mathrm{Na}]^{+}\right)$.

2-氧代- $2 H$-苯并吡喃-7-基( $E$ )-3-(4-溴苯基)丙烯酸 酯 $\left(\mathbf{D}_{6}\right)$ : 淋洗剂: 石油醚/乙酸乙酯/二氯甲烷 $(V: V$ : $V=5: 1: 1$ (400 mL). 白色固体, 产率 64.6\%. m.p. $216 \sim 218{ }^{\circ} \mathrm{C}$ (Lit. ${ }^{[32]}$ m.p. $217 \sim 218{ }^{\circ} \mathrm{C}$ ); ${ }^{1} \mathrm{H}$ NMR $(500$ $\left.\mathrm{MHz}, \mathrm{CDCl}_{3}\right) \delta: 7.83(\mathrm{~d}, J=16.0 \mathrm{~Hz}, 1 \mathrm{H}, \mathrm{CH}=\mathrm{CH}), 7.71$ $(\mathrm{d}, J=9.6 \mathrm{~Hz}, 1 \mathrm{H}, \mathrm{CH}=\mathrm{C}), 7.60 \sim 7.55(\mathrm{~m}, 2 \mathrm{H}, \mathrm{C} 4$, C6-ArH), 7.52 (d, $J=8.4 \mathrm{~Hz}, 1 \mathrm{H}, \mathrm{C} 16-\mathrm{ArH}), 7.47$ (d, $J=$ $2.1 \mathrm{~Hz}, 1 \mathrm{H}, \mathrm{C} 1-\mathrm{ArH}), 7.46$ (d, $J=1.9 \mathrm{~Hz}, 1 \mathrm{H}, \mathrm{C} 3-\mathrm{ArH})$, 7.20 (d, $J=2.1 \mathrm{~Hz}, 1 \mathrm{H}, \mathrm{C} 13-\mathrm{ArH}), 7.14$ (dd, $J=8.4,2.2$ $\mathrm{Hz}, 1 \mathrm{H}, \mathrm{C} 17-\mathrm{ArH}), 6.62(\mathrm{~d}, J=16.0 \mathrm{~Hz}, 1 \mathrm{H}, \mathrm{CH}=\mathrm{CH})$, 6.41 (d, $J=9.5 \mathrm{~Hz}, 1 \mathrm{H}, \mathrm{C} 20-\mathrm{ArH}) ;{ }^{13} \mathrm{C}$ NMR $(126 \mathrm{MHz}$, $\left.\mathrm{CDCl}_{3}\right) \delta: 164.4,160.4,154.8,153.3,146.3,142.9,132.8$, $132.4,129.8,128.6,125.5,118.4,117.1,116.7,116.1$, 110.5; IR (KBr) v: 2924, 1723, 1615, 1404, 1268, 1122, 986, 847, 812, $600 \mathrm{~cm}^{-1}$; MS (70 eV) m/z: 393.33 ([M+ $\mathrm{Na}]^{+}$).

2-氧代- $2 H$-苯并吡喃-7-基 (E)-3-(4-三氟甲基苯基) 丙烯酸酯 $\left(\mathrm{D}_{7}\right)$ : 淋洗剂: 石油醚/乙酸乙酯/二氯甲烷 $(V$ : $V: V=5: 1: 1(400 \mathrm{~mL})$. 白色固体, 产率 $45.3 \%$. m.p. $178 \sim 181{ }^{\circ} \mathrm{C}$ (Lit. ${ }^{[32]}$ m.p. $\left.178 \sim 180{ }^{\circ} \mathrm{C}\right) ;{ }^{1} \mathrm{H}$ NMR $(500$ $\left.\mathrm{MHz}, \mathrm{CDCl}_{3}\right) \delta: 7.91(\mathrm{~d}, J=16.0 \mathrm{~Hz}, 1 \mathrm{H}, \mathrm{CH}=\mathrm{CH}), 7.71$ (d, $J=10.9 \mathrm{~Hz}, 5 \mathrm{H}, \mathrm{CH}=\mathrm{C}, \mathrm{C} 1, \mathrm{C} 4, \mathrm{C} 3, \mathrm{C} 6-\mathrm{ArH}), 7.53$ (d, $J=8.4 \mathrm{~Hz}, 1 \mathrm{H}, \mathrm{C} 16-\mathrm{ArH}), 7.21$ (d, $J=2.2 \mathrm{~Hz}, 1 \mathrm{H}$, C13-ArH), 7.15 (dd, $J=8.4,2.2 \mathrm{~Hz}, 1 \mathrm{H}, \mathrm{C} 17-\mathrm{ArH}$ ), 6.71 $(\mathrm{d}, J=16.0 \mathrm{~Hz}, 1 \mathrm{H}, \mathrm{CH}=\mathrm{CH}), 6.42(\mathrm{~d}, J=9.6 \mathrm{~Hz}, 1 \mathrm{H}$,
C20-ArH); ${ }^{13} \mathrm{C}$ NMR (126 MHz, $\left.\mathrm{CDCl}_{3}\right) \delta: 164.2,160.4$, $154.8,153.2,145.7,142.9,137.2,128.7,128.6,126.1$, 126.1, 126.1, 126.0, 119.1, 118.4, 116.8, 116.2, 110.5; IR (KBr) $v$ : 2924, 1727, 1619, 1411, 1328, 1270, 1198, 1068, 985, 864, $830 \mathrm{~cm}^{-1}$; MS $(70 \mathrm{eV}) \mathrm{m} / z: 382.73\left([\mathrm{M}+\mathrm{Na}]^{+}\right)$.

2-氧代- $2 \mathrm{H}$-苯并吡喃-7-基 $(E)$-3-(4-羟基苯基)丙烯 酸酯 $\left(\mathbf{D}_{8}\right)$ : 淋洗剂: 石油醚/乙酸乙酯/二氯甲烷 $(V: V$ : $V=5: 1: 1(500 \mathrm{~mL})$. 白色固体, 产率 $43.0 \%$. m.p. 242 $244{ }^{\circ} \mathrm{C}$ (Lit. ${ }^{[32]}$ m.p. $242 \sim 244{ }^{\circ} \mathrm{C}$ ); ${ }^{1} \mathrm{H}$ NMR $(500$ MHz, DMSO- $\left.d_{6}\right) \delta: 10.17$ (s, $\left.1 \mathrm{H}, \mathrm{OH}\right), 8.10$ (dd, $J=9.6$, $0.7 \mathrm{~Hz}, 1 \mathrm{H}, \mathrm{CH}=\mathrm{CH}), 7.84 \sim 7.77(\mathrm{~m}, 2 \mathrm{H}, \mathrm{CH}=\mathrm{C}$, C1-ArH), $7.70 \sim 7.65$ (m, 2H, C4, C6-ArH), 7.36 (d, $J=$ $2.3 \mathrm{~Hz}, 1 \mathrm{H}, \mathrm{C} 16-\mathrm{ArH}), 7.23(\mathrm{dd}, J=8.4,2.2 \mathrm{~Hz}, 1 \mathrm{H}$, C17-ArH), $6.88 \sim 6.82(\mathrm{~m}, 2 \mathrm{H}, \mathrm{C} 3, \mathrm{C} 13-\mathrm{ArH}), 6.66$ (d, $J=$ $16.0 \mathrm{~Hz}, 1 \mathrm{H}, \mathrm{CH}=\mathrm{CH}), 6.49(\mathrm{~d}, J=9.6 \mathrm{~Hz}, 1 \mathrm{H}$, C20-ArH); ${ }^{13} \mathrm{C}$ NMR (126 MHz, DMSO) $\delta: 165.4,161.0$, $160.3,154.6,153.6,148.0,144.4,131.4,129.8,125.4$, 119.2, 117.1, 116.4, 116.0, 113.0, 110.7; IR (KBr) v: 3424, 2924, 1736, 1598, 1389, 1128, $817 \mathrm{~cm}^{-1}$; MS (70 eV) $\mathrm{m} / \mathrm{z}$ : $330.98\left([\mathrm{M}+\mathrm{Na}]^{+}\right)$.

2-氧代- $2 H$-苯并吡喃-7-基 $(E)$-3-(4-羟基-3-甲氧基苯 基)丙烯酸酯 $\left(\mathbf{D}_{9}\right)$ : 淋洗剂: 石油醚/乙酸乙酯/二氯甲烷 $(V: V: V=5: 1: 1(450 \mathrm{~mL})$. 白色固体, 产率 $59.3 \%$. m.p. $214 \sim 216{ }^{\circ} \mathrm{C}$ (Lit. ${ }^{[32]}$ m.p. $213 \sim 215{ }^{\circ} \mathrm{C}$ ); ${ }^{1} \mathrm{H}$ NMR $\left(500 \mathrm{MHz}, \mathrm{DMSO}-d_{6}\right) \delta: 9.77(\mathrm{~s}, 1 \mathrm{H}, \mathrm{OH}), 8.11(\mathrm{~d}, J=0.7$ $\mathrm{Hz}, 1 \mathrm{H}, \mathrm{CH}=\mathrm{CH}), 7.81(\mathrm{~d}, J=5.1 \mathrm{~Hz}, 1 \mathrm{H}, \mathrm{CH}=\mathrm{C}), 7.79$ (d, $J=2.3 \mathrm{~Hz}, 1 \mathrm{H}, \mathrm{C} 1-\mathrm{ArH}), 7.45(\mathrm{~d}, J=2.0 \mathrm{~Hz}, 1 \mathrm{H}$, C4-ArH), 7.36 (d, $J=2.2 \mathrm{~Hz}, 1 \mathrm{H}, \mathrm{C} 17-\mathrm{ArH}), 7.24$ (dd, $J=$ 8.4, $2.2 \mathrm{~Hz}, 2 \mathrm{H}, \mathrm{C} 6, \mathrm{C} 13-\mathrm{ArH}), 6.84(\mathrm{~d}, J=8.2 \mathrm{~Hz}, 1 \mathrm{H}$, C16-ArH), $6.75(\mathrm{~d}, J=15.9 \mathrm{~Hz}, 1 \mathrm{H}, \mathrm{CH}=\mathrm{CH}), 6.49(\mathrm{~d}$, $J=9.6 \mathrm{~Hz}, 1 \mathrm{H}, \mathrm{C} 20-\mathrm{ArH}), 3.84$ (s, $\left.3 \mathrm{H}, \mathrm{OCH}_{3}\right) ;{ }^{13} \mathrm{C} \mathrm{NMR}$ (126 MHz, DMSO) $\delta: 165.4,160.3,154.6,153.6,150.5$, $148.5,148.3,144.4,129.8,125.8,124.4,119.2,117.1$, $116.1,116.0,113.3,112.0,110.6,56.2$; IR (KBr) $v: 3433$, 2924, 1709, 1607, 1420, 1232, 1128, 983, $849 \mathrm{~cm}^{-1}$; MS $(70 \mathrm{eV}) \mathrm{m} / z: 359.65\left([\mathrm{M}+\mathrm{Na}]^{+}\right)$.

\section{3 抑制酪氨酸酶活性实验}

参考 Ielo 等 ${ }^{[33]}$ 的研究方法并稍作修改, 合成的衍生 物、香豆素、肉桂酸及曲酸均用 DMSO 溶解, 并稀释成 不同浓度的化合物. 将 $130 \mu \mathrm{L}$ 磷酸缓冲溶液 $(50 \mathrm{mmol} \cdot$ $\left.\mathrm{L}^{-1}, \mathrm{pH}=6.8\right), 10 \mu \mathrm{L}$ 酪氨酸酶溶液(终浓度 $33.3 \mathrm{U} / \mathrm{mL}$ ) 和 $10 \mu \mathrm{L}$ 待测化合物混匀后, 加入 $50 \mu \mathrm{L} L$-Dopa(终浓度 $0.5 \mathrm{mmol} \cdot \mathrm{L}^{-1}$ ) 混匀, 于 $490 \mathrm{~nm}$ 波长下测定 $\mathrm{OD}$ 值的变化 率. 抑制率 $(\%)=\left[\left(\mathrm{OD}_{1}-\mathrm{OD}_{2}\right) / \mathrm{OD}_{1}\right] \times 100 \%$, 其中 $\mathrm{OD}_{1}$ 为空白 $\mathrm{OD}$ 值的变化率, $\mathrm{OD}_{2}$ 为测试化合物 $\mathrm{OD}$ 值的变 
化率.

\section{4 酶抑制动力学研究}

测定方法与上述测定化合物的酪氨酸酶抑制活性 的方法类似。

酶抑制机理: $L$-Dopa 的终浓度为 $0.5 \mathrm{mmol} / \mathrm{L}$, 酪氨 酸酶的终浓度为 $25.0,33.3,41.6,50.0 \mathrm{U} / \mathrm{mL}$ 时, 测定不 同浓度化合物 $\mathbf{C}_{\mathbf{8}}$ 和 $\mathbf{D}_{\mathbf{8}}$ 在 $490 \mathrm{~nm}$ 波长下 $\mathrm{OD}$ 值的变化 率. 以不同浓度化合物作用下 $\mathrm{OD}$ 值的变化率与酶浓度 作图 ${ }^{[10]}$.

酶抑制类型: $L$-多巴的终浓度为 $0.5,0.75,1,1.25$ $\mathrm{mmol} \cdot \mathrm{L}^{-1}$, 酪氨酸酶的终浓度为 $33.3 \mathrm{U} / \mathrm{mL}$ 下, 测定不 同浓度化合物 $\mathbf{C}_{\mathbf{8}}$ 和 $\mathbf{D}_{\mathbf{8}}$ 在 $490 \mathrm{~nm}$ 波长下测定 $\mathrm{OD}$ 值的 变化率. 以 $\mathrm{OD}$ 值的变化率与多巴浓度用双倒数曲线作 图 $^{[32]}$.

\section{5 分子对接实验}

用 Sybyl-2.1.1 软件模拟化合物 $\mathbf{C}_{\mathbf{8}}$ 和 $\mathbf{D}_{\mathbf{8}}$ 与酪氨酸酶 的结合. 化合物 $\mathbf{C}_{\mathbf{8}}$ 和 $\mathbf{D}_{\mathbf{8}}$ 加氢后, 加电荷, 使用 $\mathrm{MM} 2$ 程 序进行能量最小化运算. 酪氨酸酶的晶体结构(PDB ID: 2 Y9X)进行去水, 加氢, 修复侧链酰胺, 使用酪氨酸酶 2Y9X 自带配体的活性口袋, 默认模式下与能量最小化 的化合物 $\mathbf{C}_{8}$ 和 $\mathbf{D}_{8}$ 进行对接.

\section{References}

[1] Xu, Y.; Lei, P.; Ling, Y.; Wang, S. W.; Yang X. L. Chin. J. Org. Chem. 2014, 34, 1118 (in Chinese).

(徐炎, 雷鹏, 凌云, 王圣文, 杨新玲, 有机化学, 2014, 34, 1118.)

[2] Wu, L. D.; Rathi, B.; Chen, Y.; Wu, X. H.; Liu, H.; Li, J. C.; Ming, A. J.; Han, G. RSC $A d v$. 2018, 69, 39529.

[3] Rainer, B.; Revoltella, S.; Mayr, F.; Moesslacher, J.; Scalfari, V.; Kohl, R.; Waltenberger, B.; Pagitz, K.; Siewert, B.; Schwaiger, S.; Stuppner, H. Eur. J. Med. Chem. 2019, 184, 111738.

[4] Rosada, B.; Bekier, A.; Cytarska, J.; Plazinski, W.; Zavyalova, O.; Sikora, A.; Dzitko, K.; Laczkowski, K. Z. Eur. J. Med. Chem. 2019, 184, 111765.

[5] Chen, J. M.; Li, Q. L.; Ye, Y. L.; Huang, Z. Y.; Ruan, Z. P.; Jin, N. Spectrochim. Acta, A 2020, 226, 117642.

[6] Lee, B.; Moon, K. M.; Lim, J. S.; Park, Y.; Kim, D. H.; Son, S.; Jeong, H. O.; Kim, D. H.; Lee, E. K.; Chung, K. W.; An, H. J.; Chun, P.; Seo, A. Y.; Yang, J. H.; Lee, B. S.; Ma, J. Y.; Cho, W. K.; Moon, H. R.; Chung, H. Y. Oncotarget 2017, 8, 91481.

[7] Hwang, Y. S.; Oh, S. W.; Park, S. H.; Lee, J.; Yoo, J. A.; Kwon, K.; Park, S. J.; Kim, J.; Yu, E.; Cho, J. Y.; Lee, J. Oxid. Med. Cell. Longevity 2019, 9827519.

[8] Raza, H.; Abbasi, M. A.; Aziz-ur-Rehman.; Siddiqui, S. Z.; Hassan, M.; Abbas, Q.; Hong, H.; Shah, S. A. A.; Shahid, M.; Seo, S. Y. Bioorg. Chem. 2020, 94, 103445.

[9] Cai, P. G.; Xiong, Y.; Yao, Y.; Chen, W.; Dong, X. W. New J. Chem. 2019, 43, 14102.

[10] Pillaiyar, T.; Manickam, M.; Namasivayam, V. J. Enzyme Inhib. Med. Chem. 2017, 32, 403.
[11] Ho, Y. S.; Wu, J. Y.; Chang, C. Y. Antioxidants 2019, 8, 474.

[12] Arrowitz, C.; Schoelermann, A. M.; Mann, T.; Jiang, L. I.; Weber, T.; Kolbe, L. J. Invest. Dermatol. 2019, 139, 1691.

[13] Paudel, P.; Seong, S. H.; Wagle, A.; Min, B. S.; Jung, H. A.; Choi, J. S. Food Chem. 2020, 309, 125739.

[14] Zhang, L.; Zhao, X.; Tao, G. J.; Chen, J.; Zheng, Z. P. Food Chem. 2017, 223,40 .

[15] Micheloni, O. B.; Farroni, A. E.; Garcia, P.; Furlan, R. L. E. Food Chem. 2018, 269, 638 .

[16] Wang, R.; Chai, W. M.; Yang, Q.; Wei, M. K.; Peng, Y. Y. Bioorg. Med. Chem. 2016, 24, 4620.

[17] Bhosle, M. R.; Khillare, L. D.; Mali, J. R.; Sarkate, A. P.; Lokwani, D. K.; Tiwari, S. V. New J. Chem. 2018, 42, 18621.

[18] Dinparast, L.; Hemmati, S.; Zengin, G.; Alizadeh, A. A.; Bahadori, M. B.; Kafil, H. S.; Dastmalchi, S. ChemistrySelect 2019, 4, 9211.

[19] Santi, M. D.; Bouzidi, C.; Gorod, N. S.; Puiatti, M.; Michel, S.; Grougnet, R.; Ortega, M. G. Bioorg. Chem. 2019, 82, 241.

[20] Saeedi, M.; Eslamifar, M.; Khezri, K. Biomed. Pharmacother. 2019, 110, 582.

[21] Taofiq, O.; Heleno, S. A.; Calhelha, R. C.; Fernandes, I. P.; Alves, M. J.; Barros, L.; Gonzalez-Paramas, A. M.; Ferreira, I.; Barreiro, M. F. Microchem. J. 2019, 147, 469.

[22] Tavares-da-Silva, E. J.; Varela, C. L.; Pires, A. S.; Encarnacao, J. C.; Abrantes, A. M.; Botelho, M. F.; Carvalho, R. A.; Proenca, C.; Freitas, M.; Fernandes, E.; Roleira, F. M. F. Bioorg. Med. Chem. 2016, 24, 3556.

[23] Zhao, Z. F.; Liu, G. X.; Meng, Y. F.; Tian, J. L.; Chen, X. F.; Shen, M. L.; Li, Y. X.; Li, B. Y.; Gao, C.; Wu, S. P.; Li, C. Q.; He, X. R.; Jiang, R.; Qian, M. C.; Zheng, X. H. Bioorg. Chem. 2019, 93, 103316.

[24] Zhang, X. B.; Ma, H. Y.; Sun, T. D.; Lei, P.; Yang, X. L.; Zhang, X. M.; Ling, Y. Chin. J. Org. Chem. 2019, 39, 2965 (in Chinese). (张学博, 马航宇, 孙腾达, 雷鹏, 杨新玲, 张晓鸣, 凌云, 有机 化学, 2019, 39, 2965.)

[25] Hussain, M. I.; Syed, Q. A.; Khattak, M. N. K.; Hafez, B.; Reigosa, M. J.; El-Keblawy, A. Biologia 2019, 74, 863.

[26] Kurt, Z. B.; Sonmez, F.; Ozturk, D.; Akdemir, A; Angeli, A; Supuran, C. T. Eur. J. Med. Chem. 2019, 183, 111702.

[27] Menezes, J. C. J. M. D. S.; Diederich, M. F. Eur. J. Med. Chem. 2019, 182, 111637

[28] Qiao, L. L.; Wei, Y.; Hao, S. H. Chin. J. Org. Chem. 2018, 38, 509 (in Chinese) (乔丽丽, 魏绝, 郝双红, 有机化学, 2018, 38, 509.)

[29] Xu, X. T.; Chen, J.; Lin, Z. Q.; Li, D. L.; Zhang, K.; Sheng, Z. J.; Wang, S. H.; Zhu, S.; Asiri, A. Chin. J. Org. Chem. 2019, 39, 2958 (in Chinese).

(徐学涛, 陈洁, 林芷晴, 李冬利, 张焜, 盛钊君, 王少华, 朱顺, Abdullah M. Asiri，有机化学, 2019, 39, 2958.)

[30] Wang, S. H.; Zhang, B. H.; Chen, J.; Zheng, Y. Y.; Feng, N.; Ma, A. J.; Xu, X. T.; Asiri, A. Chin. J. Org. Chem. 2020, 40, 15 (in Chinese).

(王少华, 张帮红, 陈洁, 郑䒯䒯, 冯娜, 马爱军, 徐学涛, Abdullah M. Asiri, 有机化学, 2020, 40, 15.)

[31] Xu, L.; Wang, S. H.; Li, H. Chin. J. Org. Chem. 2015, 35, 1559 (in Chinese).

(徐力, 王少华, 李辉, 有机化学, 2015, 35, 1559.)

[32] Xu, X. T.; Deng, X. Y.; Chen, J.; Liang, Q. M.; Zhang, K.; Li, D. L.; Wu, P. P.; Zheng, X.; Zhou, R. P.; Jiang, Z. Y.; Ma, A. J.; Chen, W. H.; Wang, S. H. Eur. J. Med. Chem. 2019, 189, 112013.

[33] Zhong, Y. Y.; Yu, L. J.; He, Q. Y.; Zhu, Q. Y.; Zhang, C. G.; Cui, X. P.; Zheng, J. X.; Zhao, S. Q. ACS Appl. Mater. Interfaces 2019, 11, 32769 . 